\title{
«Medizin statt Bürokratie!» - konstruktiv und konkret
}

\section{Marcel Marti}

lic. phil. hist., Leiter Politik und Kommunikation / stv. Geschäftsführer VSAO

Die zweite Welle der VSAO-Kampagne «Medizin statt Bürokratie!» rollt an. Unter dem Motto «konstruktiv und konkret» zeigen Beispiele aus der Praxis, wie sich die administrative Belastung der Spitalärztinnen und -ärzte reduzieren lässt. Zudem geht bald eine neue Themen-Webseite online. Und: Der VSAO wird mit seinem Anliegen im Bundeshaus anklopfen.

Vor genau einem Jahr fiel der Startschuss für die Kampagne «Medizin statt Bürokratie!». Sie richtete sich in der ersten Etappe vor allem an die Leitungen der Spitäler und Weiterbildungsstätten. Das Ziel: sensibilisieren. Mit leichtem Augenzwinkern illustrierte ein Leiterspiel die bürokratischen Hürden für Ärzte und Ärztinnen auf dem Weg ans Krankenbett. Zusätzlich informierte eine Broschüre über Lösungsansätze, die den Anteil der Bürotätigkeiten am ärztlichen Dienst verringern - zum unmittelbaren Nutzen der Patienten und auch der Finanzen. Denn weniger Administration heisst weniger Kosten.

Bei den Lösungen setzt nun die Fortsetzung an. "Aufgrund einer Umfrage in unseren Sektionen haben wir

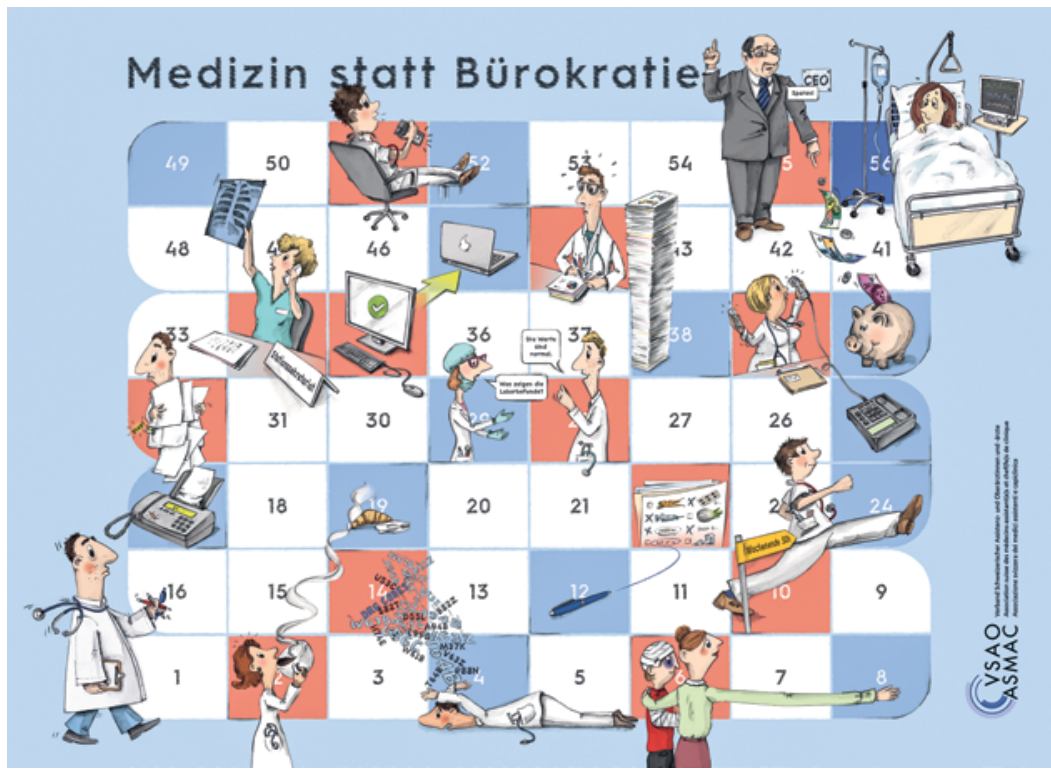

Die Fortsetzung der VSAO-Kampagne ist visuell sofort erkennbar. Sie basiert auf den Figuren und Illustrationen der ersten Welle, wie sie unter anderem beim Leiterspiel Verwendung fanden. drei Spitäler herausgegriffen, um konkret aufzuzeigen, dass man etwas tun kann», erklärt VSAO-Präsidentin Anja Zyska. Was getan wird, ist verschieden. «Deshalb zum Auftakt der zweiten Kampagnenwelle mal diese drei Beispiele; stellvertretend für viele andere, die es sicher gibt.» Man freue sich auf weitere Rückmeldungen, «da wir in einem nächsten Schritt den Katalog von möglichen, weil schon umgesetzten Massnahmen erweitern und diese propagieren wollen", so Zyska.

\section{Hôpital du Jura: Es gewinnen alle}

Das erste Beispiel stammt aus dem Jura. Angeregt durch die Kampagne entstand im Kantonsspital mit der lokalen VSAO-Sektion eine Arbeitsgruppe zum Thema «Medizin statt Bürokratie!». Derzeit läuft ein erster Test: Das Sekretariat der Abteilung Innere Medizin wurde reorganisiert, damit seine Mitarbeitenden zusätzliche Aufgaben übernehmen und die Ärzteschaft entlasten können. «Etwa beim Diktieren», präzisiert Spitaldirektor Thierry Charmillot: «Die Assistentin prüft die auf Band gesprochenen Krankenberichte zum weiteren Vorgehen. Bei Bedarf ruft sie auch den Hausarzt der Patientin an, um fehlende Informationen im Dossier zur bisherigen Behandlung einzuholen.» Das Hôpital du Jura bietet in der Inneren Medizin jedes Jahr zirka 55 Weiterbildungsplätze an. Nicht zuletzt für die Schaffung der Arbeitsgruppe ist es kürzlich vom VSAO mit der Spitalrose ausgezeichnet worden. Direktor Charmillot weist darauf hin, dass die finanziellen Rahmenbedingungen für Verbesserungen schwierig seien. Aber von den Veränderungen profitierten alle: «Unseren jungen Ärzten werden Bürden abgenommen, und für die Mitarbeitenden im Sekretariat wird das Aufgabenspektrum interessanter.» 


\section{Spital Thun: "reduce to the max»}

Szenenwechsel, Spital Thun im Kanton Bern. Dort steht die administrative Belastung seit längerem auf der Agenda, namentlich in der Medizinischen Klinik. Am Ball ist eine Arbeitsgruppe mit dem Namen "reduce to the max". Sie setzt sich aus der Leitung der Medizinischen Klinik (zwei leitende Ärzte) sowie zwei Vertretern der Assistenzärztinnen und -ärzte zusammen. Fallweise hinzu kommen Chefarzt Armin Stucki und sein Stellvertreter. "Wir holen über die Assistenzärzte regelmässig Verbesserungsvorschläge ein und schauen gemeinsam, ob und falls ja: wie sich diese realisieren lassen», erläutert Stucki. «Darüber hinaus haben wir beschlossen, getroffene Massnahmen regelmässig zu überprüfen.»

Der Chefarzt schildert ein Beispiel, das sich in der Praxis bereits bewährt hat: Wenn eine Assistenzärztin Unterlagen von extern anfordern muss, kann sie sich diese neu per E-Mail ins Sekretariat übermitteln lassen. Dort werden die Dokumente direkt ins Informationssystem der Klinik eingespiesen. «Der Umweg zuerst über die Ärztin entfällt - und sie gewinnt wertvolle Zeit für ihre eigentliche Arbeit.»

Ein anderes Thema ist die Patientenbetreuung, für die es in der Medizinischen Klinik spezielle Coaches gibt. Dabei handelt es sich um diplomierte Pflegefachfrauen, welche frühzeitig die Entlassung planen, Gespräche koordinieren und den Informationsfluss gewährleisten. Es geht um die Optimierung der Schnittstellen zwischen Arzt, Pflege und Patient. Wichtig sei, ergänzt Armin Stucki, «dass die Coaches zusammen mit den Assistenzärztinnen auf der Abteilung sind. Dadurch besteht ein enger Kontakt und Austausch, auch bei der Chefarztvisite und entsprechenden Entscheiden.» Im Weiteren führt die Klinik Qualitätszirkel durch, um Projekte interdisziplinär zu bearbeiten und sie an Anlässen allen Mitarbeitenden vorzustellen. $\mathrm{Zu}$ nennen ist etwa die Vereinfachung von Rezepten und das Erfassen der Medikamente beim Spitaleintritt.

\section{Spital Thusis: Kampf den Zeitfressern}

Korrespondenz:

Marcel Marti

VSAO

Bollwerk 10

Postfach

$\mathrm{CH}-3001$ Bern

Tel. 0313504482 (direkt)

marti[at]vsao.ch und Tag», führt Spitaldirektor Reto Keller aus. Bei der Analyse stachen die unzähligen Schnittstellen, die häufigen Rückfragen sowie mehrere Genehmigungs-, Prüf- und Nacharbeitsschleifen ins Auge. So dauerte die Erstellung eines Austrittsberichts bis zur Freigabe 13,5 Tage - das Verfassen selber hingegen nur 40 Minuten.

«Bei den Änderungen lag uns am Herzen, dass das Ganze nicht in ein IT-Projekt ausartet.»

«Wir haben daher die Wege visualisieren, Informationsflüsse aufzeichnen, Bestände zählen und das Geschehen beobachten lassen», berichtet Reto Keller. «Bei den Änderungen lag uns dann am Herzen, dass das Ganze nicht in ein IT-Projekt ausartet. Bis auf die Erneuerung respektive Digitalisierung der Diktaphone wollten wir deshalb keine neuen Systeme einführen, sondern die vorhandene Infrastruktur effizienter und sinnvoller nutzen.» Zur Zeitersparnis wurden ausserdem die Abläufe organisatorisch vereinfacht und die Anzahl Schnittstellen reduziert.

\section{Von Gutem noch besser berichten}

Mehr über die drei Fallbeispiele ist ab Mitte September auf einer neuen Kampagnen-Webseite zu erfahren. «Unsere Verbands-Homepage soll zwar voraussichtlich im nächsten Jahr ohnehin ein frisches, zeitgemässes Gesicht erhalten», erzählt VSAO-Präsidentin Anja Zyska. «Aber wir möchten der Kampagne bereits jetzt mehr Gewicht geben und über Gutes noch besser berichten.» Zyska denkt dabei auch an den im Rahmen der Herbstsession geplanten Auftritt im Bundeshaus. Zu Einzelheiten lässt sie sich noch nicht in die Karten blicken. Doch sie verrät, dass die Aktion darauf abziele, den Parlamentsmitgliedern die Problematik mit Situationen aus der Praxis vor Augen zu führen. «Schliesslich können sie als Patientinnen und Patienten ebenfalls von den negativen Folgen von zu viel Bürokratie statt mehr Zeit für den Menschen betroffen sein.»

Bei sämtlichen Aktivitäten während der zweiten Kampagnenwelle prüft der VSAO die Vernetzung mit anderen Organisationen im Gesundheitswesen. Denn konstruktiv heisst nicht zuletzt Hand in Hand mit Partnern, wo dies sinnvoll ist. Grundsätzliche Absprachen dazu sind bereits getroffen.

Bildnachweis

unikum Graphic Design/VSAO 